\title{
Congenital adrenal hyperplasia and hypokalaemic paralysis
}

\author{
S Sabapathippillai ${ }^{1}, K_{\text {Seneviratne }}{ }^{1}$, M K Ragunathan ${ }^{1}$
}

Ceylon Medical Journal 2011; 56: 169-170

\section{Introduction}

Although acute onset hypokalaemic paralysis is an uncommon cause of acute weakness, morbidity and mortality may occur due to respiratory failure and cardiac arrhythmias. Therefore it is important for a physician to have an idea regarding the underlying causes and its prompt management. To the best of our knowledge, this is the first case of congenital adrenal hyperplasia (11 beta hydroxylase deficiency) associated with hypokalaemic paralysis to be reported.

\section{Case report}

A 17-year old female, diagnosed to have congenital adrenal hyperplasia at birth (11 beta hydroxylase deficiency) came with acute onset weakness of both legs which had progressed over two days and on admission she was almost bed bound. There was no history of diarrhoea or fever. She did not have urinary or bowel incontinence. She had mild weakness of both upper limbs. There were no similar episodes in the past and no family history of a similar problem.

On examination she was afebrile, conscious, without any wasting or fasiculations and with a Glasgow coma scale of $15 / 15$. Power in the upper limbs was grade four and reflexes were diminished. In the lower limbs, power was zero with no sensory impairment and reflexes were absent. Full blood count, serum calcium, serum phosphate and serum magnesium were normal. The only abnormal finding was serum potassium of $1.8 \mathrm{mmol} / \mathrm{l}$ which was confirmed by repeated testing. Intravenous potassium replacement was done (60 mmol $\mathrm{KCl}$ over 24 hours with normal saline). On second day, serum potassium increased to $2.8 \mathrm{mmol} / \mathrm{l}$ and then to $4.3 \mathrm{mmol} / \mathrm{l}$. By second day, her power improved considerably and by day three, power and tone in the limbs were normal.

The diagnosis of hypokalaemic paralysis should be suspected in any patient with sudden onset areflexic pure motor weakness involving one or more limbs without alteration in conscious level or sphincter function and laboratory evidence of hypokalaemia [1]. The cardinal laboratory feature is serum potassium $<3.5 \mathrm{mmol} / \mathrm{l}$. Hypokalaemia and paralysis occur either due to an acute shift of potassium into the cells or due to a large deficit of potassium [2]. Symptomatology results from increased ratio between intracellular and extra cellular potassium concentration which modifies membrane polarisation and thereby alters the function of excitable tissues nerve and muscle [1]

Hypokalaemic paralysis represents a heterogenous group of disorders, which leads to a final common pathway presenting as acute weakness and hypokalaemia [3]. Hypokalaemic paralysis can be divided into two major groups $[3,4]$. The first group include patients with hypokalaemic periodic paralysis which is due to an acute shift of potassium into the cells, e.g. thyrotoxic periodic paralysis, familial periodic paralysis, hypernatraemic hypokalaemic paralysis and sporadic periodic paralysis.

The second group consists of patients presenting with hypokalaemic paralysis which is due to potassium depletion, e.g. mineralocorticoid excess (adrenal adenoma, adrenal hyperplasia, licorice ingestion), renal tubular acidosis, diuretics and magnesium depletion.

\footnotetext{
${ }^{1}$ Teaching Hospital, Karapitiya, Galle, Sri Lanka.
}

Correspondence: SS, e-mail: <saumy1@yahoo.com>. Received 8 October 2010 and revised version accepted 25 March 2011. Competing interests: none declared. 
Primary hyperaldosteronism (Conn's syndrome) presenting as hypokalaemic paralysis has been reported [1]. Hypokalaemic paralysis is known to occur in 10-15\% of patients with primary aldosteronism [4]. In congenital adrenal hyperplasia (CAH) due to 11 beta hydroxylase deficiency (rare form), hypertension and hypokalaemia complicated with seizures and arrhythmias has been described in a nine year old girl, originally diagnosed to have 21 hydroxylase deficiency which later turned out to be 11 beta hydroxylase deficiency. It occurred after abrupt withdrawal of oral dexamethasone and continuation of fludrocortisones [5].

Our patient presented with hypertension, hypokalaemia and acute paralysis and at presentation she was on nifedipine, hydrocortisone and spironolactone. Excess mineralocorticosteroid action might play an important role in the pathogenesis of hypokalaemic paralysis [6]. It was supported by previous evidence that paralysis can be induced by administration of mineralocorticoids and by ACTH [6]. In CAH (11 beta hydroxylase deficiency) increased blood pressure and hypokalaemia occur due to impaired conversion of 11 deoxycorticosterone to corticosterone resulting in accumulation of 11 deoxycorticosterone, a potent mineralocorticoid. Our patient was not on any other drugs apart from nifedipine, hydrocortisone and spironolactone. She was on physiological replacement dose of hydrocortisone. Hydrocortisone can cause hypokalaemia via its inorganic metabolism (mineralocortcoid effect) but in clinical practice this is rare [7]. Even though she was on potassium retaining spironolactone, she presented with hypokalaemic paralysis which is uncommon.

\section{References}

1. Ahlawat SK, Sachdev A. Hypokalaemic paralysis. Postgraduate Medical Journal 1999; 75: 193-4.

2. Lin SH, Lin YF, Chu P, et al. Laboratory tests to determine the cause of hypokalemia and paralysis. Archives of Internal Medicine 2004; 164: 1561-6.

3. Stedwell RE, Allen KM, Binder LS. Hypokalemic paralysis: a review of aetiologies, pathophysiology, presentation and therapy. American Journal of Emergency Medicine 1992; 10: $143-6$.

4. Lin SH, Lin YF, Halperin ML. Hypokalaemia and paralysis. Quarterly Journal of Medicine 2001; 94: 133-9.

5. Chang SH, Lee HH, Way PJ, et al. Congenital adrenal hyperplasia with 11 beta hydroxylase deficiency. Journal of Formos Medical Association 2004; 103: 860-4.

6. Streeten DHP, Speller PJ. The role of mineralocorticoids in the pathogenesis of hypokalaemic periodic paralysis. Journal of Clinical Endocrinology Metabolism 1974; 39: 326-33.

7. Bennett PN, Laurence DR. Clinical Pharmacology, 10th edition, Churchill Livingstone 1992: 550-1. 